\title{
Atenção integral à hipertensão arterial e diabetes mellitus: implementação da Linha de Cuidado em uma Região de Saúde do estado de São Paulo, Brasil
}

I 1 Sonia Isoyama Venancio, ${ }^{2}$ Tereza Etsuko da Costa Rosa,

${ }^{3}$ Ana Aparecida Sanches Bersusa I

Resumo: Objetivou-se avaliar a implementação da Linha de Cuidado (LC) em hipertensão arterial e diabetes mellitus em uma Região de Saúde do estado de São Paulo. Trata-se de uma pesquisa avaliativa desenvolvida em três etapas: caracterização das regiōes para seleção da região-caso; entrevistas com gestores e profissionais de saúde que atuam na Atenção Básica, Média e Alta Complexidade e entrevistas com pacientes em Ambulatórios Médicos de Especialidades. A análise baseou-se em um modelo lógico que considera a influência da macro e micropolítica na construção da LC. De modo geral, insuficiências foram identificadas nas dimensões da macropolítica (organização da rede de serviços e utilização de protocolos) e micropolítica (coordenação do cuidado pela $\mathrm{AB}$, vinculação e responsabilização do cuidador). Conclui-se que, apesar dos avanços alcançados, grandes desafios se impõem para o alcance da integralidade da atenção à hipertensão arterial e diabetes mellitus, sendo necessários investimentos nos processos da macro e micropolítica.

> Palavras-chave: hipertensão; diabetes mellitus; atenção integral à saúde; atenção primária à saúde; serviços de saúde.

\author{
1 Instituto de Saúde, Secretaria \\ de Estado de Saúde. São \\ Paulo-SP, Brasil (soniav@isaude. \\ sp.gov.br). \\ 2 Instituto de Saúde, Secretaria \\ de Estado de Saúde. São Paulo- \\ SP, Brasil (tererosa@isaude. \\ sp.gov.br). \\ ${ }^{3}$ Instituto de Saúde, Secretaria \\ de Estado de Saúde. São \\ Paulo-SP, Brasil (anabersusa@ \\ ig.com.br).
}

Recebido em: 12/03/2015 Aprovado em: 20/07/2015 


\section{Introdução}

As Linhas de Cuidado (LC) podem ser definidas como o conjunto de saberes, tecnologias e recursos necessários ao enfrentamento de determinados riscos, agravos ou condições específicas do ciclo de vida ou de outro critério médicosanitário, a ser ofertado de forma oportuna, articulada e contínua pelo sistema de saúde. Sua implementação é estratégia central para a organização e a qualificação das Redes de Atenção à Saúde (RAS) com vistas à integralidade da atenção (BRAGA, 2006). Nessa perspectiva, o usuário passa a ser o elemento estruturante de todo processo de produção da saúde (FRANCO; MAGALHÃES JUNIOR. 2004). Para o alcance da integralidade sistêmica (MATTOS, 2006), a atenção básica $(\mathrm{AB})$ deve ser resolutiva e se articular com os demais níveis de atenção à saúde, de média e alta complexidade.

O Decreto no 7.508, de 28 de junho de 2011 (BRASIL, 2011), reafirma a importância da construção das RAS no contexto de consolidação do Sistema Único de Saúde (SUS), a fim de constituir malhas que interconectem e integrem os estabelecimentos e serviços de saúde de determinado território, organizando-os sistematicamente para que diferentes níveis e densidades tecnológicas de atenção estejam articulados e adequados para o atendimento ao usuário e para a promoção da saúde. Ressalta-se que a importância da construção de redes se dá, entre outros, pelo aumento da prevalência de doenças crônicas não transmissíveis, pelo maior potencial de avanços na integralidade e na construção de vínculos e pelos custos crescentes no tratamento das doenças (SILVA, 2011). Segundo o Vigitel (Sistema de Monitoramento de Fatores de Risco e Proteção para Doenças Crônicas Não Transmissíveis por intermédio de entrevistas telefônicas), implantado pela Secretaria de Vigilância em Saúde (SVS) do Ministério da Saúde e realizado em 2011, na população de São Paulo na faixa de idade igual ou maior de 18 anos, a prevalência de pessoas que referiram serem portadores de diabetes para o conjunto de municípios foi de 5,9\%; a de hipertensão foi de 22,53\% (BRASIL, 2012a).

Frente à transição demográfica e epidemiológica em curso no Brasil, torna-se urgente a articulação de políticas e programas voltados para o enfrentamento 
dessa realidade, bem como o aprofundamento sobre o cuidado que tem sido

dispensado aos portadores de doenças crônicas não transmissíveis nos serviços de saúde (MALTA et al., 2006).

No estado de São Paulo, a implementação de LC em hipertensão arterial e diabetes mellitus foi inserida como ação prioritária no Plano Estadual de Saúde (2012-2015). Para tal, o Estado conta com a maior capacidade instalada vinculada ao SUS no país, com 4,5 mil Unidades Básicas de Saúde (UBS), 1,5 mil ambulatórios de especialidade e 582 hospitais distribuídos pelo Estado. O desafio que se impõe é a garantia do acesso universal dos pacientes portadores desses agravos e da integralidade da atenção, por meio da articulação dos serviços nos di ferentes níveis de complexidade (SÃO PAULO, 2012).

Nesse contexto, o presente estudo objetivou avaliar a implementação da LC em hipertensão arterial e diabetes mellitus em uma Região de Saúde do estado de São Paulo.

\section{Métodos}

Realizou-se uma pesquisa avaliativa que, por tratar de um objeto complexo, tornou necessária a combinação de métodos quantitativos e qualitativos (PATTON, 1997). Optou-se pela utilização do estudo de caso (YIN, 2001), que propicia a realização de uma investigação em profundidade, considerandose como unidade de análise uma Região de Saúde do estado de São Paulo. Tomou-se também como premissa, para a avaliação da LC, a técnica da condição traçadora (ou condição marcadora), que utiliza problemas ou agravos específicos de saúde para obtenção de dados que serão usados para avaliar a qualidade dos serviços de saúde (SAMICO; FIGUEIRÓ; FRIAS, 2010) No presente estudo, a escolha da hipertensão e da diabetes mellitus se justifica pela magnitude dessas doenças, por sua crescente morbimortalidade, pelos custos que agregam e por gerarem eventos contínuos/crônicos que, com frequência, se agudizam, especialmente quando não bem cuidados, podendo ser um tema analisador das situações de fragmentação da LC (MALTA; MERHY, 2010). O modelo de avaliação é apresentado na Figura 1: 


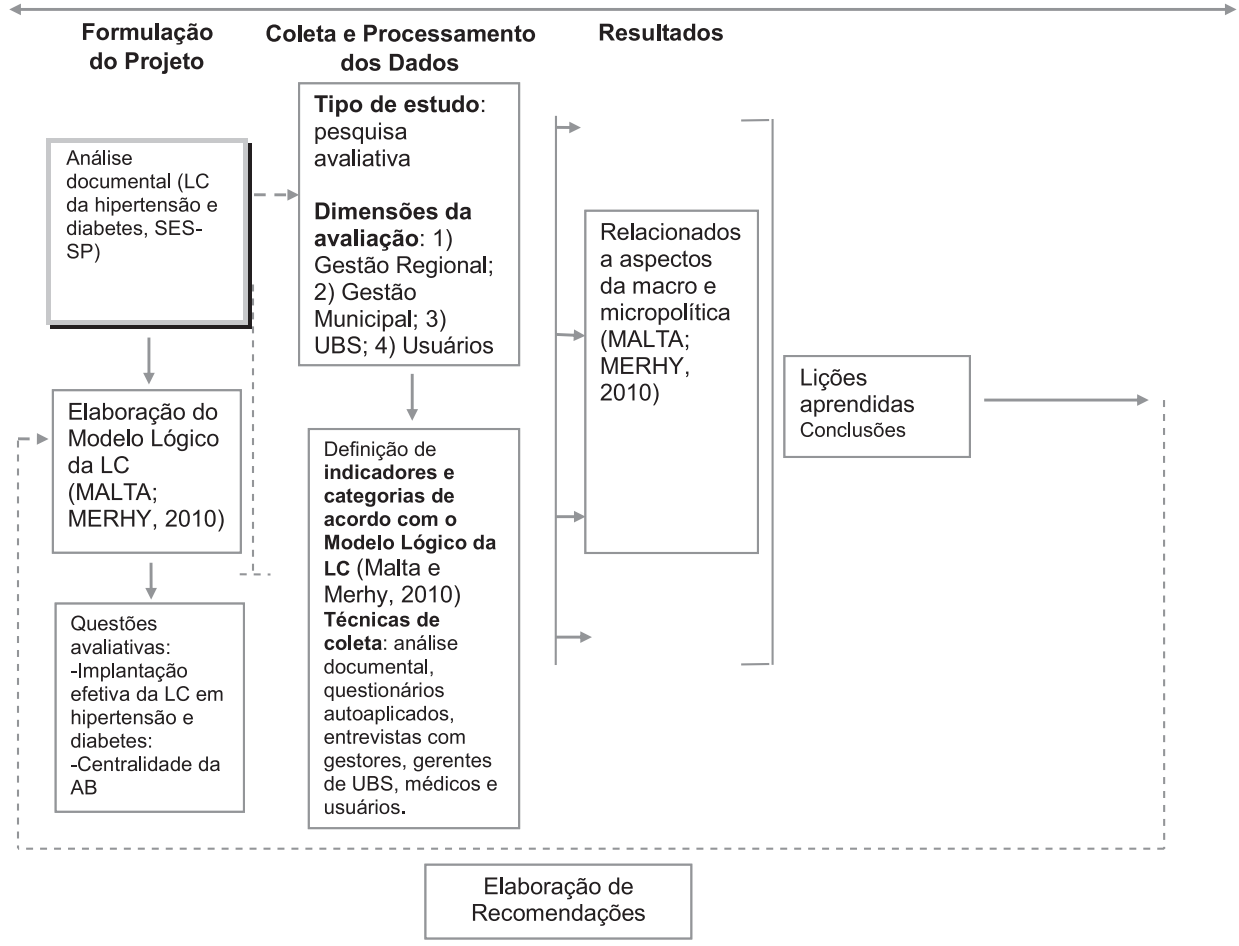

\section{Definição do caso: Região de Saúde e municípios}

A primeira etapa do estudo consistiu na seleção de uma Região de Saúde. Para tal, realizou-se um levantamento de dados secundários para caracterização das 17 Regiōes de Saúde do estado. Levaram-se em consideração: o Índice Paulista de Responsabilidade Social (IPRS) (FUNDAÇÃ̂ SEADE, 2010); o percentual da população com acesso à saúde suplementar; a cobertura da Estratégia Saúde da Família (ESF); a cobertura de consultas básicas e especializadas; as taxas de mortalidade por acidente vascular cerebral (AVC) e internaçôes por diabetes mellitus; e a presença de Ambulatórios Médicos de Especialidades (AME) e hospitais terciários de referência na Região.

Com base nessas informaçôes e nas discussões realizadas com a Coordenadoria de Regiōes de Saúde da SES-SP, a região selecionada foi a de Taubaté (DRS XVII) por ter vários municípios (61\%) em condições piores 
de desenvolvimento humano (IPRS 4 e 5), percentual de população com acesso à saúde suplementar inferior à media do Estado (34,3\%); cobertura da ESF (35\%) que possibilitaria a comparação entre UBS com os diferentes modelos de atenção; cobertura de consultas básicas $(1,6)$ e especializadas $(0,7)$ inferiores às coberturas do Estado; taxas de mortalidade por AVC e internações por diabetes mellitus superiores às médias estaduais (9,3 e 76,6 por $100 \mathrm{mil}$ habitantes, respectivamente); e pela existência de dois AME, o que viabilizaria a avaliação da contribuição desse novo equipamento de saúde na região. A Região de Saúde de Taubaté é composta por 39 municípios subdivididos em quatro Colegiados de Gestão Regional (CGR): Vale do Paraíba e Região, Alto Vale do Paraíba, Circuito da Fé e Vale e Litoral.

A etapa seguinte consistiu da seleção de uma amostra intencional de municípios para a realização da coleta de dados. Essa seleção foi feita mediante a análise da população dos CGR, número de UBS nos municípios, cobertura municipal da ESF, existência e número de AME, número de ambulatórios de especialidades, número de hospitais gerais e existência de hospitais de referência. A análise desses dados indicou ser adequado incluir os quatro municípios polos dos CGR (São José dos Campos, Guaratinguetá, Caraguatatuba e Taubaté) por possibilitarem a análise de diferentes realidades e a comparação entre os modelos da ESF e AB tradicional. A apresentação/pactuação dessa proposta foi feita em uma reunião da macrorregião de Saúde, organizada pelo DRS XVII com a presença de gestores regionais e municipais.

A terceira etapa consistiu em um levantamento das UBS de cada município polo dos CGR. Em seguida, foi realizado um sorteio aleatório, considerando duas tipologias de UBS segundo o modelo de atenção: unidades básicas com equipes de saúde da família/programa de agentes comunitários de saúde (UBSF/ PACS) ou UBS tradicionais, de forma que no conjunto das UBS se obtivesse uma amostra contemplando em torno de $50 \%$ de UBSF/PACS e $50 \%$ de UBS tradicionais.

\section{Coleta de dados}

A coleta dos dados visou à obtenção de informações sobre a implantação da LC a partir da percepção dos diferentes atores envolvidos no processo: gestor 
regional, gestores municipais, gerentes de UBS, médicos de UBS, médicos especialistas e pacientes que aguardavam atendimento em ambulatórios de especialidades dos municípios envolvidos.

Foram elaborados nove instrumentos de coleta de dados, pré-testados em serviços de saúde de um dos municípios da região: questionários semiestruturados, autoaplicados pelos gestores municipais e aplicados aos demais atores por entrevistadoras previamente treinadas, acompanhadas por uma supervisora de campo. A coleta de dados foi realizada no período de 09/11/2011 a 30/05/2012

Os dados foram digitados em bancos de dados no programa Epilnfo e analisados no programa Stata 10.0.

\section{Análise dos dados}

O plano de análise baseou-se no modelo lógico apresentado na figura 2, construído a partir da perspectiva proposta por Malta e Merhy (2010), sobre a influência da macro e micropolítica na construção da LC das doenças crônicas não transmissíveis.

Figura 2. Modelo Lógico da LC em doenças crônicas não transmissíveis

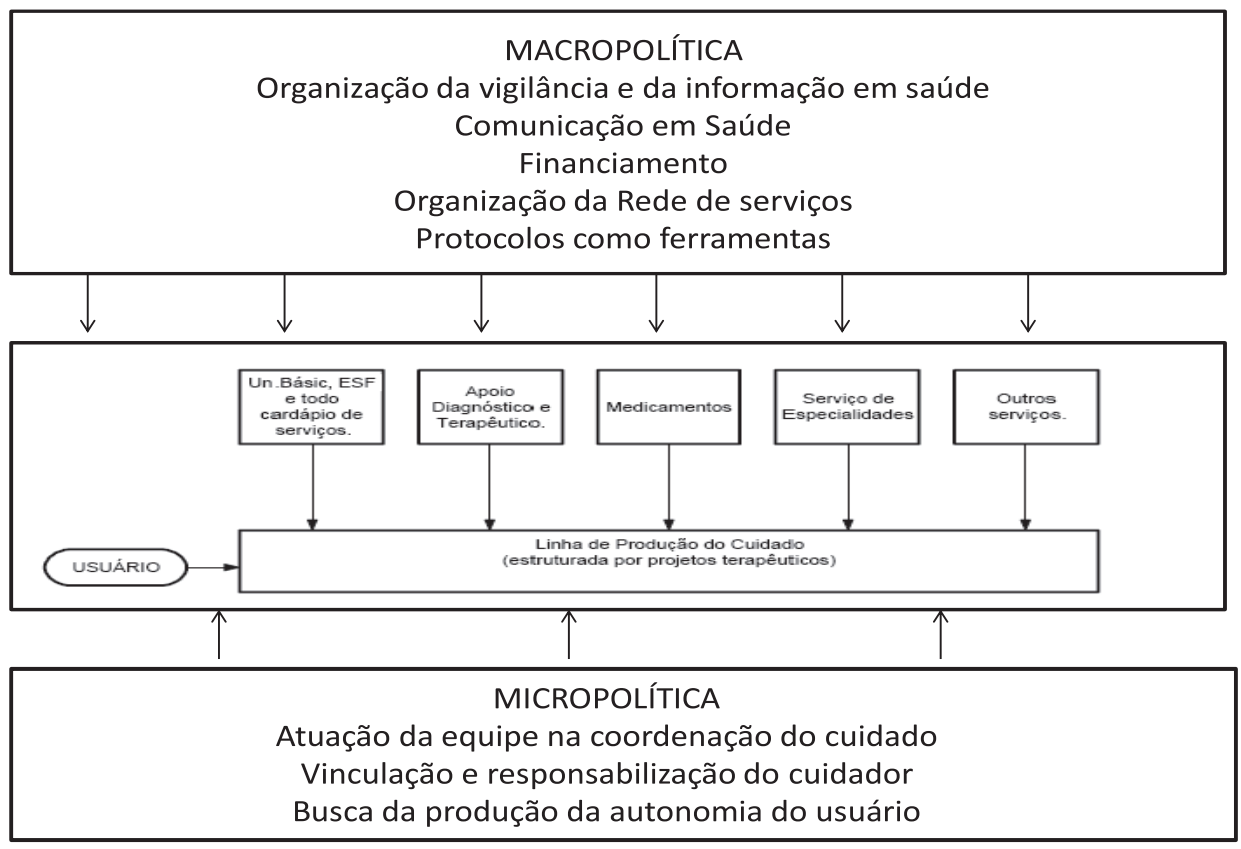


Inicialmente, foram definidas categorias de análise, relacionadas às perspectivas da macro e micropolítica. Em seguida, foram construídos indicadores a partir dos dados empíricos coletados, baseados em uma ou mais fontes de informação. Nesse sentido, foi possível realizar a triangulação dos dados a partir da percepção de diferentes atores, propiciando maior possibilidade de aproximação da realidade local.

O projeto foi aprovado pelo Comitê de Ética do Instituto de Saúde sob o protocolo $n^{\circ}$ 009/2011 e as entrevistas foram aplicadas mediante a assinatura do Termo de Consentimento Livre Esclarecido.

\section{Resultados}

No tocante às categorias relacionadas à dimensão da macropolítica (Quadro 1), apresentam-se os resultados obtidos junto ao gestor regional, 20 gestores municipais (que representam $51 \%$ do universo dos municípios da região), gerentes das 41 UBS visitadas, 38 médicos entrevistados por ocasião dessas visitas e 417 pacientes que aguardavam atendimento em ambulatórios da média complexidade (estaduais ou municipais). 


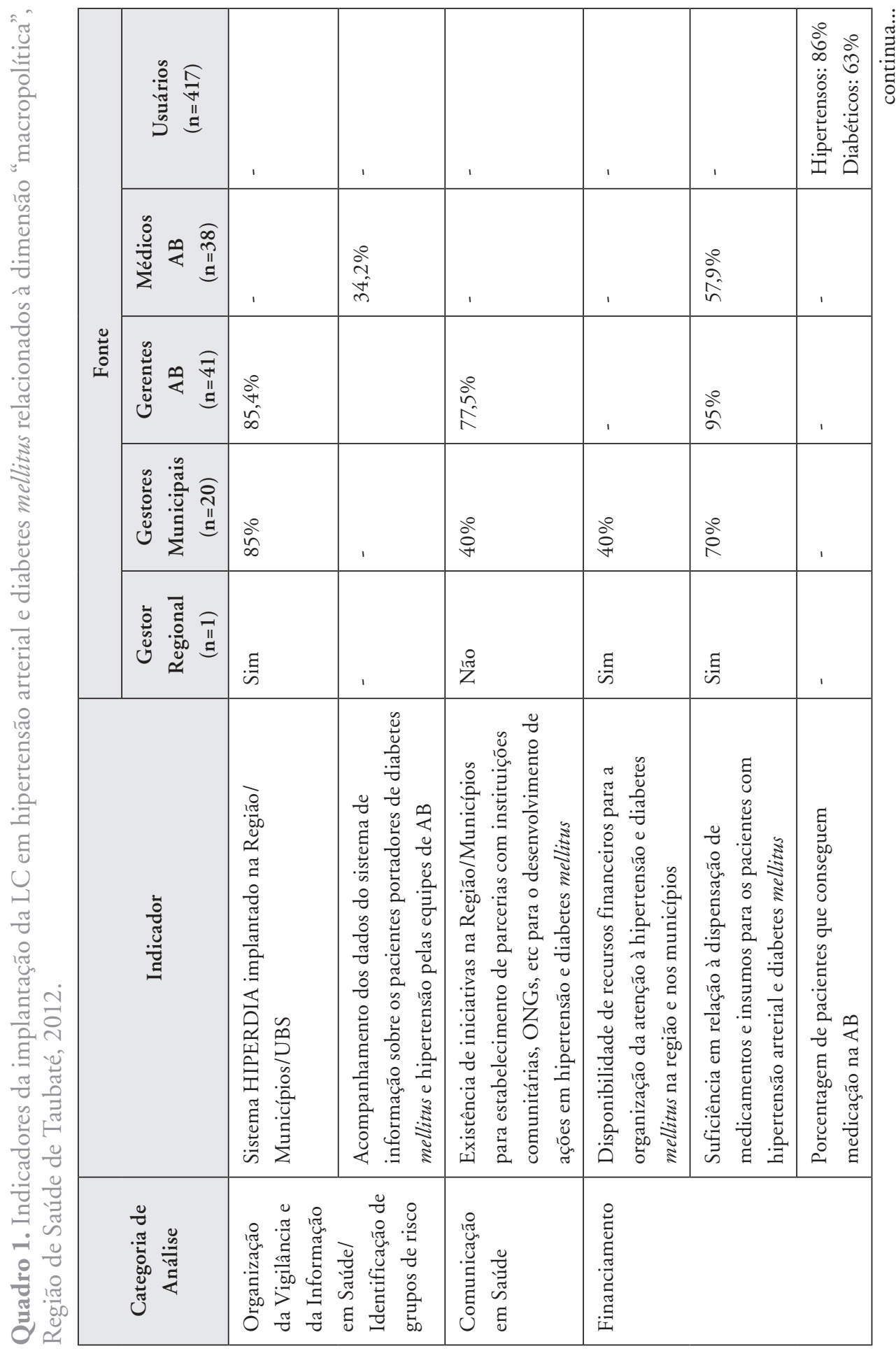




\begin{tabular}{|c|c|c|c|c|c|c|c|}
\hline \multirow{5}{*}{ 䒕 } & 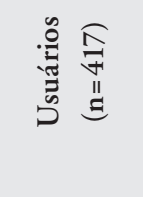 & ' & ' & ' & 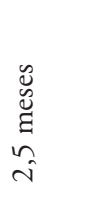 & ' & I \\
\hline & 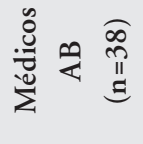 & . & ' & $\begin{array}{l}\stackrel{0}{ } \\
\stackrel{\vec{F}}{\vec{F}}\end{array}$ & I & $\begin{array}{l}\stackrel{0}{\infty} \\
\infty \\
\tilde{n}\end{array}$ & $\stackrel{\circ}{\stackrel{9}{a}}$ \\
\hline & 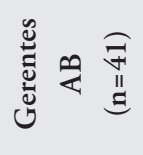 & $\begin{array}{l}\stackrel{0}{ } \\
\stackrel{\circ}{0}\end{array}$ & $\stackrel{\stackrel{0}{\sim}}{\underset{\hbar}{n}}$ & $\begin{array}{l}\stackrel{0}{A} \\
\stackrel{-}{=}\end{array}$ & ' & $\begin{array}{l}\stackrel{\circ}{+} \\
\stackrel{\sigma}{0}\end{array}$ & $\stackrel{\stackrel{\circ}{d}}{\stackrel{+}{d}}$ \\
\hline & 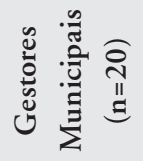 & $\begin{array}{l}\stackrel{0}{0} \\
\stackrel{\infty}{0}\end{array}$ & $\stackrel{\stackrel{\circ}{n}}{\stackrel{n}{n}}$ & $\begin{array}{l}\stackrel{0}{n} \\
\stackrel{n}{n}\end{array}$ & I & $\begin{array}{l}\stackrel{\circ}{+} \\
\stackrel{\sigma}{0}\end{array}$ & $\begin{array}{l}\dot{0}^{\circ} \\
\text { in }\end{array}$ \\
\hline & 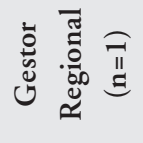 & 施 & 坣 & $\Xi$ & & 坣 & $\Xi$ \\
\hline & 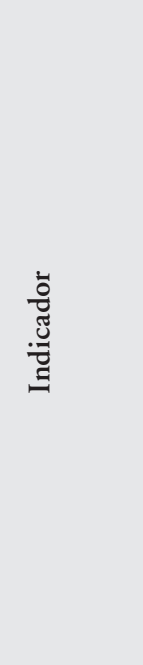 & 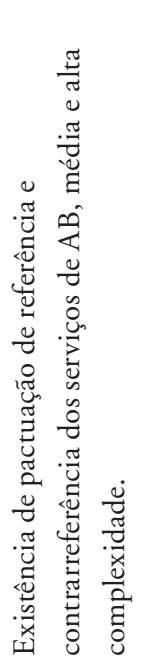 & 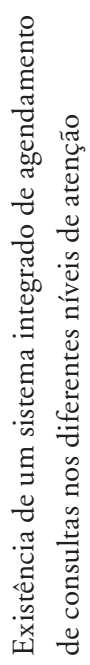 & 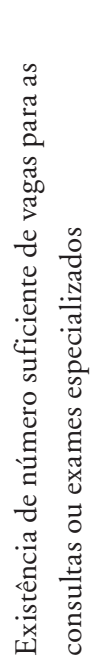 & 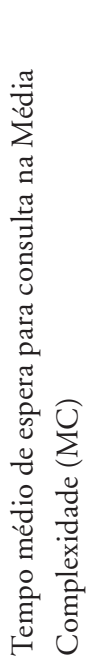 & 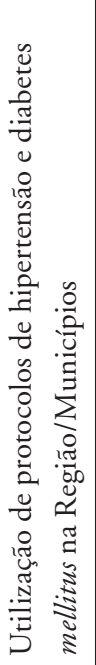 & 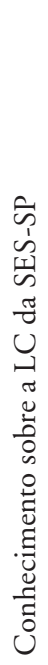 \\
\hline & 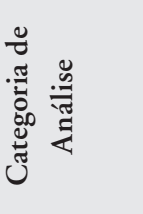 & 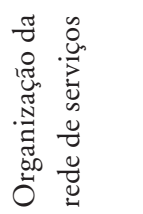 & & & & $\begin{array}{ll}0 & \\
0 & \\
0 & \\
0 & 0 \\
0 & 0 \\
0 & 0 \\
0 & 0\end{array}$ & 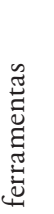 \\
\hline
\end{tabular}


Sobre a organização da vigilância e da informação em saúde, embora o gestor regional e a maioria dos gestores municipais tenham a percepção de que o sistema de gestão clínica de hipertensão arterial e diabetes mellitus da AB, Hiperdia, está implantado na região, somente um terço dos médicos relatou a apropriação desses dados pelas equipes de saúde.

Em relação à existência de iniciativas na região e nos municípios para o estabelecimento de parcerias com instituições comunitárias e ONGs, a fim de desenvolver ações em hipertensão arterial e diabetes mellitus, verificou-se que as mesmas são mais frequentes nos relatos dos gerentes das UBS do que o que foi informado pelo gestor regional e gestores municipais.

A existência de sistemas integrados de agendamento de consultas nos diferentes níveis foi relatada com maior frequência pelos gestores municipais do que pelos gerentes das UBS envolvidas no estudo, sendo que essa estratégia não foi relatada no nível regional.

Quanto à disponibilidade de recursos financeiros para a implantação da LC, embora o gestor regional relate suficiência de recursos, somente 40\% dos gestores municipais relataram o mesmo. Mais especificamente em relação à dispensação de medicamentos e insumos para hipertensos e diabéticos, identificou-se suficiência na percepção do gestor regional e para a maioria dos atores consultados. Segundo a informação dos pacientes entrevistados, houve disponibilidade de medicamentos na $\mathrm{AB}$ para $86 \%$ dos hipertensos e $63 \%$ dos diabéticos, indicando ainda problemas no acesso aos medicamentos da LC.

Sobre a organização da rede de atenção à saúde, houve pactuação de referência e contrarreferência na região. Porém, somente $35 \%$ dos gestores municipais, $17 \%$ dos gerentes de UBS e $41 \%$ dos médicos tiveram percepção favorável em relação à suficiência de vagas para consultas/exames na MC. Foi possível identificar, junto aos pacientes, que o tempo médio de espera para consultas em ambulatórios de especialidades foi de 2,5 meses.

$\mathrm{Na}$ última categoria relacionada à dimensão "macro", quanto à utilização de protocolos como ferramentas para a organização da atenção à saúde, verificou-se que essa prática ainda não atinge a totalidade dos municípios, sendo relatada por $63 \%$ dos gestores municipais e gerentes de UBS e por pouco mais da metade dos médicos. 
Para a dimensão micropolítica (quadro 2), apresentam-se os resultados obtidos junto aos gerentes das UBS, médicos da $A B$ e pacientes. Quanto à atuação das equipes de $\mathrm{AB}$ na coordenação do cuidado, inicialmente buscou-se identificar como se organiza o fluxo de referência para MC e contrarreferência. Verificou-se que o agendamento das consultas com especialistas pela UBS foi relatado por menos da metade dos gerentes (46\%). Todos os médicos entrevistados relataram preenchimento de ficha de encaminhamento dos pacientes para outros locais de atendimento, porém somente $41,3 \%$ e $26,0 \%$ dos gerentes $\mathrm{da} \mathrm{AB}$ e médicos, respectivamente, relataram a disponibilidade de laudos de exames ou relatórios clínicos de atendimentos realizados por especialistas. Segundo as informaçóes dos pacientes, havia compartilhamento do atendimento entre $\mathrm{AB}$ e ambulatórios de especialidades para 55\% dos pacientes atendidos nos ambulatórios municipais de especialidades e 93,5\% dos pacientes atendidos nos AME.

A seguir, descrevem-se os indicadores selecionados para a análise da vinculação e responsabilização do cuidador. Segundo informações dos gerentes, $80 \%$ das UBS trabalham com território definido e população adscrita e $61 \%$ realizam busca ativa de pacientes hipertensos e diabéticos na comunidade; contudo, somente $46 \%$ dos pacientes hipertensos e $50 \%$ dos diabéticos tiveram o diagnóstico da doença realizado pela $\mathrm{AB} .77,5 \%$ das UBS adotam procedimentos de busca ativa nos casos em que são detectadas alterações nos exames e os pacientes não retornam à unidade, e 78\% relatam a realização de visitas domiciliares. Chama atenção que percentual menor de visitas domiciliares foi relatado pelos pacientes (42\%). Quanto ao atendimento de intercorrências clínicas sem consulta previamente agendada, 95\% dos gerentes e $87 \%$ dos médicos referiram que as mesmas são atendidas nas UBS. Porém, somente $45 \%$ dos pacientes relataram procurar a UBS nos casos em que "passam mal".

No tocante à busca da produção da autonomia dos pacientes para o cuidado, verificou-se que $78 \%$ das UBS desenvolviam atividades educativas em grupos, sendo que percentual semelhante de pacientes relatou receber orientaçōes em grupo nas UBS. 

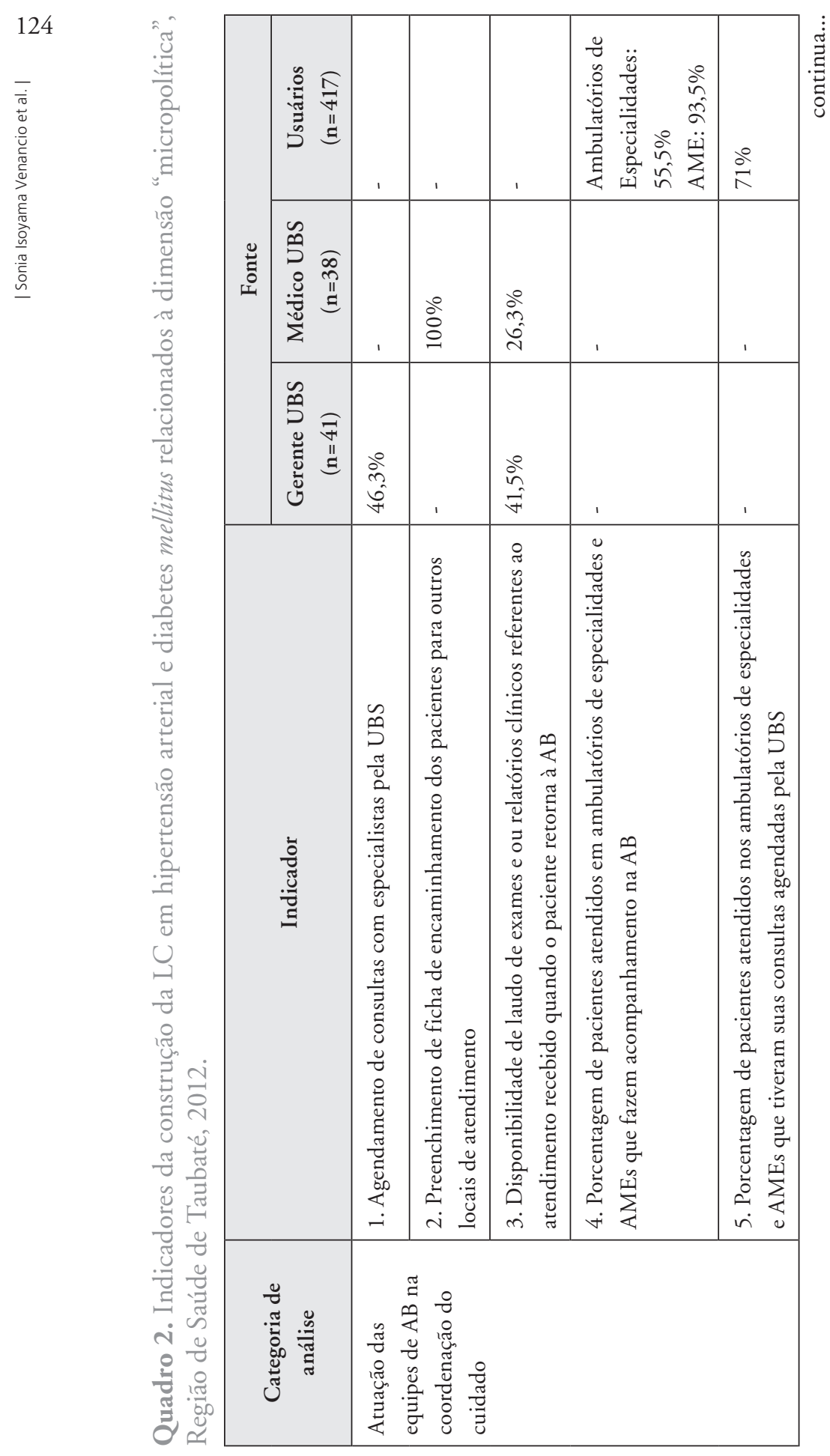


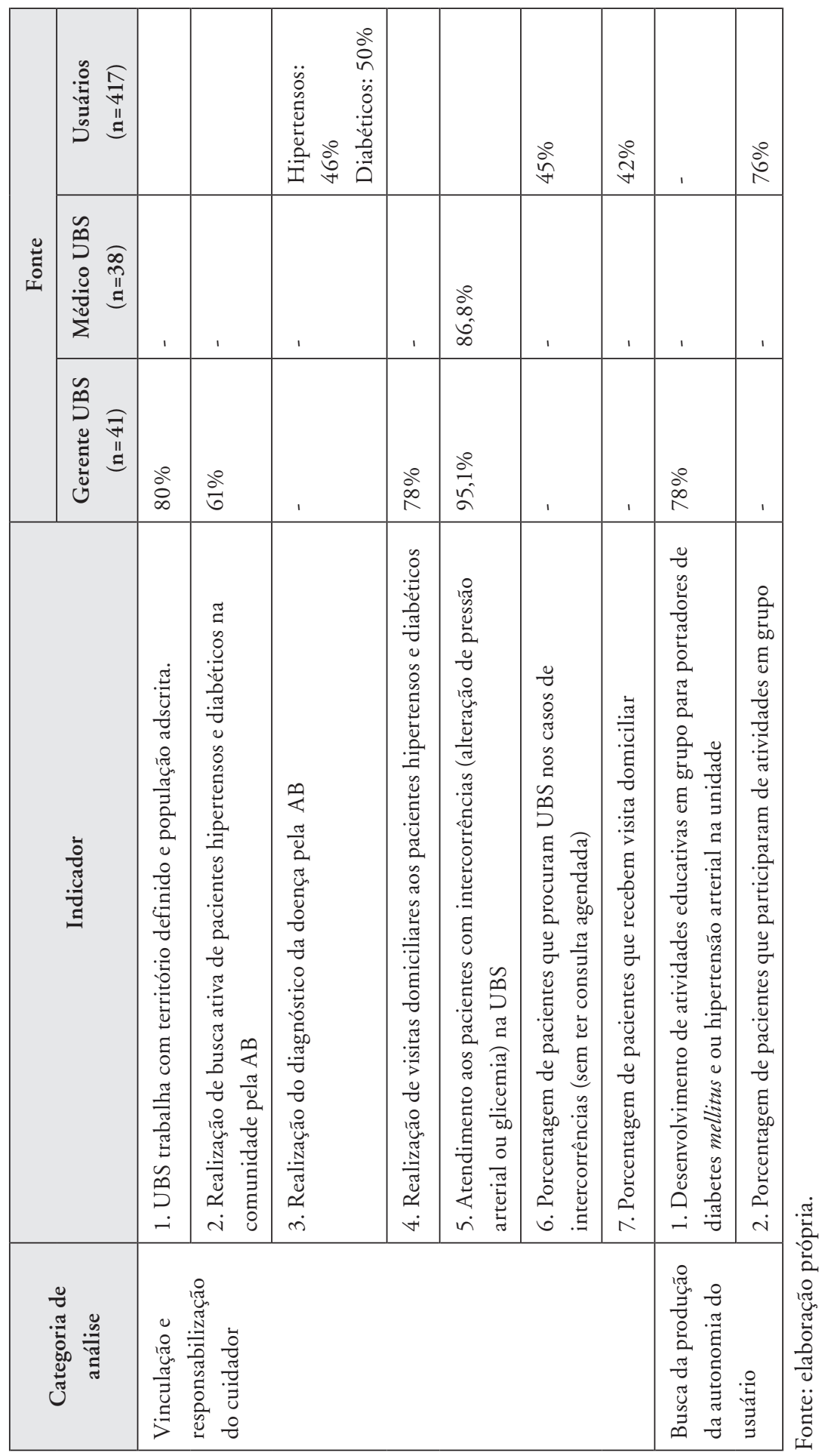




\section{Discussão}

Este estudo possibilitou a análise da implantação da LC em hipertensão arterial e diabetes mellitus, à luz do referencial proposto por Malta e Merhy (2010, p. 595), segundo o qual a produção da saúde implica formatos institucionais que articulam respostas macro e microinstitucionais, sendo que para a efetiva implantação da LC é preciso ordenar tanto demandas de organização do sistema de saúde e suas interfaces, quanto o processo de trabalho em saúde (MALTA; MERHY, 2010; CECÍLIO; MERHY, 2003).

No tocante aos macroprocessos, insuficiências foram identificadas em todas as categorias de análise selecionadas. Em relação à vigilância e informação em saúde, optou-se por analisar a implantação do sistema Hiperdia, por ser a estratégia adotada pelo MS para o cadastramento e acompanhamento de portadores de hipertensão arterial ou diabetes mellitus atendidos na rede ambulatorial do SUS, permitindo gerar informação para aquisição, dispensação e distribuição de medicamentos de forma regular e sistemática a todos os pacientes cadastrados. Embora os gestores e gerentes tenham relatado sua implantação, é interessante observar que as equipes de saúde não se apropriaram do sistema para o planejamento. Se esforços são feitos para a construção de sistemas de informação, como o Hiperdia, a sua subutilização, como a verificada neste estudo, evidencia que a sua implantação não garante que, de fato, o sistema cumpra o seu principal objetivo: identificar e dimensionar problemas individuais e coletivos para subsidiar a definição de prioridades. Outro aspecto que pode agravar tal situação é apontado por estudos que questionam a fidedignidade das informações e a adequação do preenchimento dos formulários do sistema Hiperdia (JARDIM; LEAL, 2009; ZILMER et al. 2010). Os trabalhos confirmam a ausência de mecanismos de avaliação e de controle da qualidade de dados produzidos pelos sistemas de informação de saúde no Brasil (SILVA; LAPREGA, 2005), o que acaba por legitimar a não utilização dos sistemas de informação de saúde para o planejamento e para a gestão .

São estratégias fundamentais para a efetivação da LC a disseminação de informações e a utilização de meios de comunicação visando à mobilização e engajamento da comunidade para definir açôes a serem desenvolvidas para o controle da hipertensão arterial e diabetes mellitus (BRASIL, 2006). As iniciativas nesse sentido foram ausentes no âmbito do Departamento Regional 
de Saúde e pouco frequentes no âmbito municipal. Porém, o estabelecimento

de parcerias com instituições comunitárias e ONGs foi relatado com maior intensidade pelos gerentes das UBS. Não obstante, as deficiências identificadas nesse aspecto indicam que são pequenas as ações de promoção e prevenção à saúde destinadas à população, o que não difere de outros resultados da literatura (SIQUEIRA et al., 2009; PICCINI et al. 2012). Isso merece atenção especial por parte dos gestores, destacando-se que o fornecimento de orientações sobre cuidado com o peso corporal, atividade física e dieta pobre em sal tem sido apontado como importante indicador de qualidade do cuidado em hipertensão arterial (ASCH et al., 2001) e que as baixas frequências desse tipo de orientaçôes contribuem para a perda da efetividade no manejo da doença (PICCINI et al., 2012).

Quanto à organização da rede de serviços, os gestores relataram a existência de pactuação de referência e contrarreferência na LC. Porém, estudo realizado em cinco Regiões de Saúde do Estado de São Paulo mostrou que os mecanismos formais de referenciamento são ainda insuficientes na LC da hipertensão arterial e diabetes mellitus, acarretando dificuldades para o estabelecimento dos fluxos efetivos de referência e contrarreferência (VENANCIO et al., 2011; ROSA et al., 2009), o que pode contribuir para o "estrangulamento" da MC, apontado por gestores municipais, gerentes e medicos da $A B$.

A utilização de protocolos constitui também uma estratégia para a garantia da qualidade e integralidade da atenção à saúde. Esses devem contemplar a análise de custo-efetividade, os programas e recomendações já existentes, definir insumos estratégicos e diagnósticos disponíveis e embasar-se nas melhores evidências científicas (MALTA; MERHY, 2010). Embora a SES-SP tenha se empenhado na elaboração de um protocolo voltado à hipertensão arterial e diabetes mellitus, lançado em 2010, verificou-se pouca adesão a protocolos nos municípios analisados e também um desconhecimento do material elaborado, especialmente pelos médicos da $\mathrm{AB}$.

Por fim, em relação à macropolítica, vale ressaltar que, quanto à existência de recursos financeiros para implantação da LC, verificada também por meio da disponibilidade de medicamentos e insumos para o tratamento de hipertensos e diabéticos, não foram identificadas dificuldades na percepção de todos os atores 
envolvidos. Embora tenha sido alto o percentual de pacientes que referiram ter acesso aos medicamentos de hipertensão (86\%), em relação ao diabetes mellitus, o acesso foi relatado por $63 \%$, o que demonstra dificuldades, contradizendo os relatos dos gestores e profissionais de saúde.

Passando à análise das categorias relacionadas à micropolítica, em relação à atuação das equipes da $\mathrm{AB}$ na coordenação do cuidado, optou-se por analisar o papel da equipe da $\mathrm{AB}$ na integração da rede de serviços, pois, segundo Malta e Merhy (2010, p. 599), os coordenadores da LC deverão buscar uma melhor articulação entre as várias "estações cuidadoras", a fim de garantir a integralidade da atenção. Nesse sentido, somente $43 \%$ dos gerentes entrevistados referiram que o agendamento das consultas com especialistas era realizado pelas UBS. Também foram identificadas dificuldades em relação ao acesso das equipes da $\mathrm{AB}$ aos relatórios e exames realizados na $\mathrm{MC}$, o que pode comprometer a continuidade do cuidado. Porém, situação mais favorável foi relatada pelos pacientes, uma vez que 93,5\% daqueles atendidos nos AME referiram acompanhamento na UBS, e 55,5\% daqueles que fazem seguimento de casos mais graves nos ambulatórios também relataram acompanhamento compartilhado com a AB. Além disso, $71 \%$ dos pacientes informaram que sua consulta no ambulatório de especialidades havia sido agendada pela UBS, percentual superior ao relatado pelos gerentes das UBS selecionadas. Vale ressaltar que o vínculo estabelecido entre o paciente e a unidade cuidadora é essencial para o manejo das condiçóes crônicas em tela, haja vista achados que apontam que consultar-se regularmente com o mesmo médico melhora a adesão às recomendações para o tratamento (PICCINI et al., 2012).

A vinculação e responsabilização do cuidador pressupõe a compreensão dos trabalhadores de saúde acerca das necessidades dos usuários (FEUERWERKER, 2005). Nesse sentido, verificou-se que $80 \%$ das UBS trabalhavam com território definido e clientela adscrita, conforme preconiza a Política Nacional de Atenção Básica (BRASIL, 2012b). Outras questões que podem demonstrar responsabilização por parte dos cuidadores, como a utilização de procedimentos de busca ativa de pacientes na comunidade e nos casos em que se identificam alteraçôes em exames realizados, não foram relatados pela totalidade dos gerentes das UBS. 
Embora a maioria das unidades tenha informado efetuar visitas domiciliares, a atividade parece não ter o alcance esperado, posto que menos da metade dos usuários entrevistados informou receber esse atendimento em seus domicílios. Tais constatações podem ser mais bem compreendidas com base em estudos de abordagem qualitativa. Sakata et al. (2007), por exemplo, evidenciam que a equipe de saúde compreende que as visitas domiciliares são uma ferramenta de aproximação entre profissionais e usuário, mas que o tempo para a sua realização é sempre identificado como insatisfatório por concorrer com muitas outras tarefas.

Outro estudo, de metodologia análoga, focalizando a visão do usuário sobre a visita domiciliar, obteve achados semelhantes, que dão indícios de que essa atividade é percebida como algo esporádico e que não contribui nem para melhorias na assistência profissional, nem para a organização do serviço "uma vez que [a visita] revela um não compromisso com a alteridade e com o resgate do continuum saúde-doença” (ALBUQUERQUE; BOSI, 2009, p. 1110). Esses resultados corroboram para a compreensão de que práticas como a assistência domiciliar podem contribuir na transformação dos cuidados à saúde na atenção primária, mas que ainda precisam ser incorporadas na agenda semanal dos médicos, "qualificada[s], propositiva[s] e direcionada[s] a problemas específicos" (SANTOS et al., 2012, p. 2699).

Ainda no tocante ao atendimento às necessidades dos usuários, embora a maioria dos gerentes e médicos relate o atendimento à demanda espontânea, somente $45 \%$ dos pacientes atendidos em ambulatórios de especialidades relataram procurar a UBS nos casos de intercorrências (sem agendamento prévio), o que demonstra sua baixa vinculação à $A B$. Estudo conduzido em quatro grandes centros urbanos brasileiros evidenciou que boa parte dos atendimentos de equipes de saúde da família é oriunda de demandas espontâneas da população, indicando que as unidades de $\mathrm{AB}$ à saúde também estão atentas a esse tipo de demandas (SANTOS et al., 2012). Todavia, uma análise minuciosa sobre situações que demandam utilização dos serviços na atenção primária de saúde empreendida por Santos e Penna (2013, p. 152) mostra um descompasso entre as necessidades e demandas dos usuários e o que os profissionais julgam que eles necessitam. Os usuários que buscam espontaneamente atendimentos em unidades de saúde de $A B$, geralmente, procuram por consulta médica, com encaminhamentos para 
especialistas ou para realização de exames. Entretanto, tais demandas não recebem o encaminhamento adequado, pois são interpretadas pelos profissionais de saúde como sendo equivocadas e desnecessárias.

Esses achados podem dar elementos para a compreensão do cenário evidenciado pelo presente estudo, no qual os serviços de $\mathrm{AB}$ estão acessíveis às demandas espontâneas. No entanto, o baixo volume de buscas por atendimentos nos casos de intercorrências nas UBS indica que elas não são reconhecidas como serviços de procura regular. Nesse sentido, vale salientar que capacidade de uma UBS de equilibrar os atendimentos programados e a demanda espontânea de forma adequada e resolutiva é o que vai caracterizar o serviço como de primeiro contato e de uso continuado (STARFIELD, 2002).

Por fim, consideraram-se as atividades educativas em grupos, além de facilitadoras da vinculação dos portadores à rede básica (REINERS et al. 2008; RIBEIRO et al 2012), também estratégicas para a busca da produção de autonomia dos pacientes. Nesse sentido, pode-se observar que as ações educativas grupais não são suficientemente valorizadas pela $\mathrm{AB}$, posto que não foram relatadas pela totalidade dos pacientes. Esse resultado, somado às evidências da baixa frequência de ações de promoção e prevenção anteriormente apontadas, fornece elementos para a inferência sobre a baixa efetividade da $A B$ no manejo da hipertensão e da diabetes (SIQUEIRA et al., 2009; PICCINI et al., 2012).

Os achados deste estudo, de forma geral, demonstram que apesar dos avanços alcançados no tocante à implementação da LC em hipertensão arterial/diabetes mellitus na região estudada, grandes desafios se impõem para que de fato se alcance a integralidade da atenção. A metodologia empregada possibilitou a triangulação de informações sobre a percepção de diferentes atores envolvidos no processo, além de possibilitar, por meio das entrevistas com usuários atendidos em ambulatórios de especialidades, reconstituir, ao menos em parte, o percurso realizado na $\mathrm{LC}$ desde a $\mathrm{AB}$ até a média complexidade.

É importante ressaltar que as pontes e os entrecruzamentos entre a macro e a micropolítica atravessam todo o percurso da LC (MALTA; MERHY, 2010, p. 600). São necessários investimentos em ambas as dimensões para que se alcance a integralidade da atenção. Espera-se que os resultados apresentados sejam úteis para o enfrentamento dos desafios que ainda precisam ser superados, facilitando as costuras necessárias à efetiva implementação da LC. ${ }^{1}$ 


\section{Referências}

ALBUQUERQUE, A. B. B.; BOSI, M. L. M. Visita domiciliar no âmbito da Estratégia Saúde da Família: percepções de usuários no Município de Fortaleza, Ceará, Brasil. Cad. Saúde Pública, Rio de Janeiro, v. 25, n. 5, p. 1103-1112, 2009. Disponível <http://www. scielo.br/pdf/csp/v25n5/17.pdf>. Acesso em: 30 jan. 2015.

ASCH, S. et al. A new approach for measuring quality of care for women with hypertension. Arch. Intern. Med. USA, v. 161, n. 10, p. 1329-1135, 2001. Disponível em: < http://archinte. jamanetwork.com/article.aspx?articleid=648182. Acesso em: 30 jan. 2015.

BRAGA, E. C. Critérios de suficiência para análise de redes assistenciais. Consulta Pública no 26. Exposição de Motivos. Agência Nacional de Saúde Suplementar. Rio de Janeiro, 15 de dezembro de 2006. Disponível em: <http://www.ans.gov.br/component/content/ article/52-consultas-publicas/54rede-conveniada-de-medicos-hospitais-clinicas-elaboratorios>. Acesso em: 30 jan. 2015.

BRASIL. Presidência da República Casa Civil. Subchefia para Assuntos Jurídicos. Decreto $n^{\circ}$ 7.508, de 28 de junho de 2011. Regulamenta a lei no 8.080, de 19 de setembro de 1990, o planejamento da saúde, a assistência à saúde e a articulação interfederativa. Disponível <http://www.planalto.gov.br/ccivil_03/_ato2011-2014/2011/decreto/D7508.htm>. Acesso em: 13 nov. 2012.

Ministério da Saúde. Conselho Nacional de Secretários da Saúde. Colegiado de Gestão Regional na Região de Saúde 3 intraestadual. Orientações para organização e funcionamento. Série Pactos pela Saúde 2006, v. 10. Disponível em: <http://portal.saude. gov.br/portal/arquivos/pdf/volume_10.pdf>. Acesso em: 3 fev. 2011.

Secretaria de Atenção à Saúde. Vigitel Brasil 2011. Vigilância de Fatores de Risco e Proteção para Doenças Crônicas por Inquérito Telefônico. Série G. Estatística e Informação em Saúde. Brasília. 2012a. Disponível em: <http://portalsaude.saude.gov.br/portalsaude/ arquivos/pdf/2012/Ago/22/vigitel_2011_final_0812.pdf>. Acesso em: 4 out. 2013.

. Secretaria de Atenção à Saúde. Departamento de Atenção Básica. Política Nacional de Atenção Básica/Ministério da Saúde. Secretaria de Atenção à Saúde. Departamento de Atenção Básica. - Brasília: Ministério da Saúde, 2012b. Disponível em: <http://dab.saude. gov.br/portaldab/pnab.php>. Acesso em: 30 jan. 2015.

CECÍlIO, L. C. O.; MERHY E. A integralidade do cuidado como eixo da gestão hospitalar. Campinas: Unicamp; 2003. (mimeogr.). Disponível <http://www.uff.br/saudecoletiva/ professores/merhy/capitulos-07.pdf>. Acesso em: 12 nov. 2012.

FEUERWERKER, L. Modelos tecnoassistenciais, gestão e organização do trabalho em saúde: nada é indiferente no processo de luta para a consolidação do SUS. Interface, São Paulo, v. 9, n. 18, p. 498-506, 2005. Disponível em: <http://www.scielo.br/pdf/icse/ v9n18/a03v9n18.pdf>. Acesso em: 30 jan. 2015. 
FRANCO, T. B.; MAGALHÂES JUNIOR, H. M. O. Trabalho em Saúde: olhando e experienciando o SUS no cotidiano. 2a ed. São Paulo: Hucitec, 2004.

FUNDAÇÃO SEADE. IPRS versão 2010. Metodologia. Disponível em: <http://www. seade.gov.br/projetos/iprs/ajuda/2008/metodologia_2010.pdf>. Acesso em: 15 nov. 2012. JARDIM, A. D. I; LEAL, A. M. O. Qualidade da informação sobre diabéticos e hipertensos registrada no Sistema Hiperdia em São Carlos-SP, 2002-2005. Physis, Rio de Janeiro, v. 19, n. 2, p. 405-417, 2009. Disponível em: <http://www.scielo.br/pdf/physis/v19n2/v19n2a09. pdf>. Acesso 30 jan. 2015.

MALTA, D. C.; MERHY, E. L. O percurso da linha do cuidado sob a perspectiva das doenças crônicas não transmissíveis. Interface, São Paulo, v. 14, n. 34, p. 593-605, 2010. Disponível em: < http://www.scielo.br/pdf/icse/v14n34/aop0510.pdf>. Acesso em: 30 jan. 2015.

MALTA, D. C. et al. A construção da vigilância e prevenção das doenças crônicas não transmissíveis no contexto do Sistema Único de Saúde. Epidemiol. Serv. Saude, Brasília, v. 15, n. 1, p. 47-65, 2006. Disponível em: <http://scielo.iec.pa.gov.br/pdf/ess/v15n3/ v15n3a06.pdf>. Acesso em: 30 jan. 2015.

MATTOS, R. A. Os sentidos da integralidade: algumas reflexões acerca de valores que merecem ser defendidos. In: PINHEIRO, R., MATTOS, R. A. (Org.). Os sentidos da integralidade na atenção e no cuidado à saúde. Rio de Janeiro: UERJ/IMS, Abrasco, 2006. p. 41-66.

PATTON, M. Q. Utilization-focused evaluation: process and premises. 3a ed. California: The News Century Text, Sage Publications; 1997.

PICCINI, R. X. et al. Promoção, prevenção e cuidado da hipertensão arterial no Brasil. Rev. Saúde Pública, São Paulo, v. 46, n. 3, p. 543-50, 2012. Disponível em: <http://www.scielo. br/pdf/rsp/v46n3/en_3208.pdf>. Acesso em: 30 jan. 2015.

REINERS, A. A. O. et al. Produção bibliográfica sobre adesão/não adesão de pessoas ao tratamento de saúde. Cien. \& Saúde Colet., Rio de Janeiro, v. 13, sup., p. 2299-2306, 2008. Disponível em: <http://www.scielo.br/pdf/csc/v13s2/v13s2a34.pdf>. Acesso em: 30 jan. 2015.

RIBEIRO, A. G et al. Hipertensão arterial e orientação domiciliar: o papel estratégico da saúde da família. Rev Nutr., São Paulo, v. 25, n. 2, p. 271-282, 2012. Disponível em: <http://www.scielo.br/pdf/rn/v25n2/09.pdf>. Acesso em: 30 jan. 2015.

ROSA, T. E. C. et al. Da atenção às doenças cardiovasculares e diabetes mellitus: o papel da regionalização do Sistema Único de Saúde. Rev. Bras. Epidemiol., São Paulo, v. 12, n. 2, p. 158-171, 2009. Disponível em: <http://www.scielo.br/pdf/rbepid/v12n2/06.pdf>. Acesso em: 30 jan.2015. 
SAKATA, K. N. et al. Concepções da equipe de saúde da família sobre as visitas domiciliares. Rev Bras Enf., São Paulo, v. 60, n. 6, p. 659-664, 2007. Disponível em: < http://www. scielo.br/pdf/reben/v60n6/07.pdf>. Acesso em: 30 jan. 2015.

SAMICO, I.; FIGUEIRÓ, A. C.; FRIAS, P. G. Abordagens metodológicas na Avaliação em Saúde. In: SAMICO, I. et al. (Org.). Avaliação em Saúde: Bases Conceituais e Operacionais. Rio de Janeiro: MedBook, 2010. p. 15-28.

SANTOS, A. M. et al. Práticas assistenciais das Equipes de Saúde da Família em quatro grandes centros urbanos. Cien. \& Saúde Colet. Rio de Janeiro, v. 17, n. 10, p. 2687-2702, 2012. Disponível em: <http://www.scielo.br/pdf/csc/v17n10/18.pdf>. Acesso em: 30 jan. 2015.

SANTOS, T. V. C.; PENNA, C. M. M. Demandas cotidianas na atenção primária: o olhar de profissionais da saúde e usuários. Texto \& Contexto Enferm, Santa Catarina, v. 22, n. 1, p. 149-56, 2013. Disponível em: <http://www.scielo.br/pdf/tce/v22n1/pt_18.pdf> Acesso em: 30 jan. 2015.

SÃO PAUlO. Secretaria de Estado da Saúde de São Paulo. Plano Estadual 2012-2015. São Paulo: Secretaria da Saúde, 2012. 200p. Disponível em: <http://www.saude.sp.gov.br/ resources/ses/perfil/gestor/documentos -de-planejamento-em-saude/plano-estadual-desaude-2012-2015-sessp/pes_2012_2015.pdf>. Acesso em: 30 jan. 2015.

SILVA, S. F. Organização de redes regionalizadas e integradas de atenção à saúde: desafios do Sistema Único de Saúde (Brasil). Ciên. \& Saúde Colet., Rio de Janeiro, v. 16, n. 6, p. 2753-2762, 2011. Disponível em: <http://www.scielo.br/pdf/csc/v16n6/14.pdf>. Acesso em: 30 jan. 2015.

SILVA, A. S.; LAPREGA, M. R. Avaliação crítica do Sistema de Informação da Atenção Básica (SIAB) e de sua implantação na região de Ribeirão Preto. Cad. Saúde Pública, Rio de Janeiro, v. 21, n. 6, p. 1821-1828, 2005. Disponível em: <http://www.scielo.br/pdf/csp/ v21n6/21.pdf>. Acesso em: 30 jan. 2015.

SIQUEIRA, F. V. et al. Fatores considerados pela população como mais importantes para manutenção da saúde. Rev. Saúde Pública, São Paulo, v. 43, n. 6, p. 961-971, 2009. Disponível em: <http://www.scielo.br/pdf/rsp/v43n6/07.pdf>. Acesso em: 30 jan. 2015.

STARFIELD, B. Atenção Primária: equilíbrio entre necessidades de saúde, serviços e tecnologia. Brasília: UNESCO, MS; 2002. Disponível em: <http://bvsms.saude.gov.br/ bvs/publicacoes/atencao_primaria_p1.pdf>. Acesso em: 12 mai. 2012.

VENANCIO, S. I. et al. Referenciamento regional em saúde: estudo comparado de cinco casos no Estado de São Paulo, Brasil. Cienc. \& Saúde Colet., Rio de Janeiro, v. 16, n. 9, p. 3951-3964, 2011. Disponível em: <http://www.scielo.br/pdf/csc/v16n9/a32v16n9.pdf>. Acesso em: 30 jan. 2015. 
YIN, R. K. Estudo de caso: planejamento e métodos 2a ed. Porto Alegre: Bookman, 2001. ZILMER, J. G. V. et al. Avaliação da completude das informaçōes do HIPERDIA em uma unidade básica do sul do Brasil. Rev. Gaúcha Enferm., Porto Alegre, v. 31, n. 2, p. 240-246, 2010. Disponível em: http://www.scielo.br/scielo.php?script=sci_arttext\&pid =S1983-14472010000200006. Acesso em: 30 jan. 2015 .

\section{Nota}

${ }^{1}$ S. I Venancio participou do delineamento e concepção da pesquisa, análise dos dados, elaboração do texto e da revisão final. T. E. C. Rosa participou do delineamento e concepção da pesquisa, análise dos dados, elaboração do texto e da revisão final. A. A. S. Bersusa participou do delineamento e concepção da pesquisa, análise dos dados e da revisão final. 


\section{Abstract}

Comprehensive care to hypertension and diabetes mellitus: implementation of the Care Line in a Health Region of Sao Paulo, Brazil

We aimed to evaluate the implementation of Care Line (CL) in hypertension and diabetes mellitus in a health region of the state of São Paulo. We conducted an evaluation research, combining quantitative and qualitative methods, developed in three steps: characterization of the regions for selection of region - case; interviews with managers and health professionals working in primary care, medium and high complexity levels and interviews with patients in Outpatient Medical Specialties. The analysis was based on a Logical Model considering the influence of macro and micro policy in the construction of CL. In general, weaknesses were identified in the dimensions of macro policy (public health service network and use of protocols) and micro (coordination of care by Primary Health, linking and accountability caregiver). Despite the progress, major challenges are necessary to achieve integrated care in hypertension and diabetes mellitus, and investments in micro and macro processes are necessary.

> Key words: hypertension; diabetes mellitus; comprehensive health care; primary health care; health services. 